\title{
Marijuana: An Underappreciated Risk Factor for Acute Type A Aortic Dissection?
}

\author{
Iam Claire Sarmiento, BSN, ${ }^{1}$ Ashley T. Giammarino, MS, ${ }^{2}$ S. Jacob Scheinerman, MD, ${ }^{1}$ \\ Antonio J. Guirola, ${ }^{1}$ Alan R. Hartman, ${ }^{3}$ Derek R. Brinster, MD, ${ }^{1}$ Jonathan M. Hemli, MD ${ }^{1}$ \\ ${ }^{1}$ Department of Cardiovascular and Thoracic Surgery, Lenox Hill Hospital/Northwell Health, New York, NY; \\ ${ }^{2}$ Touro College of Osteopathic Medicine, Middletown, NY; \\ ${ }^{3}$ Department of Cardiovascular and Thoracic Surgery, North Shore University Hospital/Northwell Health, New York, NY.
}

\section{ABSTRACT}

Background: Stimulants such as cocaine and amphetamines are well-established risk factors for acute aortic dissection. Despite the fact that marijuana is the most commonly used illicit drug in the United States, its relationship to acute aortic syndromes has not been well studied.

Methods: A comprehensive retrospective review was undertaken of all consecutive patients who presented with acute Stanford type A aortic dissection from January 2017 to December 2019. Of 152 patients identified, 51 (33.6\%) underwent comprehensive urine toxicology screening at clinical presentation. The characteristics and outcomes of the patients with urine results positive for tetrahydrocannabinol (THC) $(\mathrm{n}=9,17.6 \%)$ were compared with the 42 patients who had no evidence of recent marijuana consumption.

Results: Of the 51 dissection patients who underwent broad-spectrum urine toxicology screening upon presentation, $9(17.6 \%)$ returned positive results for THC, a proportion higher than would be expected for the general population. All THC patients were male; 3 concurrently tested positive for cocaine, and 3 others had evidence of recent amphetamine use. The THC patients were significantly younger than the non-THC patients (mean \pm standard deviation age $48 \pm 11.3$ versus $61.4 \pm 12.3$ years, respectively, $P=.004)$. A greater proportion of the THC cohort had a known diagnosis of aortic aneurysm before the dissection ( $44.4 \%$ versus $4.8 \%, P=.006)$. All patients underwent expeditious surgical repair. Thirty-day mortality for the entire cohort of 51 patients was $19.6 \%$ (10 deaths); for the THC group, it was $11.1 \%$ ( 1 death). There was no difference in the incidence of major postoperative complications between the 2 groups.

Conclusion: Marijuana is the third most commonly used substance in the United States, after alcohol and tobacco. Although marijuana use is understudied, our results suggest

Received October 21, 2020; received in revised form November 24, 2020; accepted November 24, 2020.

Correspondence: Fonathan M. Hemli, MD, MSc, FRACS, Department of Cardiovascular \& Thoracic Surgery, Lenox Hill Hospital, 130 E 77th St, 4th Floor, New York, NY 10075; 212-434-3000; fax: 212-434-4559 (e-mail: jhemli@nortbwell.edu). that marijuana may be a contributing risk factor for acute type A aortic dissection, particularly in patients with other predisposing risk factors. Given the recent national trend to legalize marijuana, with the concomitant potential for exponential increases in its consumption, we suggest that the diagnosis of aortic dissection be considered earlier in any younger patient who presents with suggestive symptoms, especially if there is a history of recent marijuana use.

\section{INTRODUCTION}

Within the past decade, 33 states in the United States have legalized the appropriation of medicinal marijuana, and 11 states have formally decriminalized the use of tetrahydrocannabinol (THC) for recreational purposes [ProCon.org 2020]. Consequently, and not surprisingly, there has been a steady increase in the national consumption of marijuana, especially among teens and younger adults. According to the 2018 National Survey on Drug Use and Health, 53.2 million people (approximately $19.4 \%$ of the US population) ages $\geq 12$ years admitted to some form of illicit drug use in 2018; 3.5 million people used marijuana, confirming it as the most commonly imbibed substance that year (not counting alcohol and tobacco). By comparison, cocaine use was reported by 874,000 people, and methamphetamine by 205,000 [SAMHSA 2018].

Recreational and illicit drug use has been linked to adverse cardiovascular outcomes. The association between cocaine and amphetamine consumption and increased risk for acute aortic dissection has been well described [Shah 2015; Eagle 2002; Wako 2007]. Both agents are sympathomimetic agonists, potentially exacerbating stress on the aortic wall, with a consequent increased risk of dissection or rupture. Although marijuana has been shown to impact the cardiovascular system in a number of ways, including increasing the risk for acute coronary syndromes, as well as being intrinsically arrhythmogenic [DeFilippis 2020; Richards 2019], its relationship to aortic dissection has not been thoroughly explored, a state of affairs that seems a little odd, particularly in light of its prevalent use among the population.

We sought to further examine any potential association between marijuana and acute aortic dissection, in an attempt to better define the risk of an acute aortic syndrome in the large population that consumes this substance. 


\section{METHODS}

\section{Study Population and Definitions}

A comprehensive retrospective review was undertaken of our prospectively collected database to identify all patients who underwent repair for acute Stanford type A aortic dissection from January 2017 to December 2019. Those patients without broad-spectrum urine toxicology screen upon clinical presentation were excluded from analysis. The characteristics of those patients with a urine test positive for THC were compared with those who had no evidence of marijuana consumption (no THC) (Figure 1).

Definitions of patient characteristics, perioperative variables, and postoperative outcomes were obtained from the New York State Cardiac Surgery Reporting System (https:// www.health.ny.gov/forms/cardiac_surgery) and from the Society of Thoracic Surgeons Adult Cardiac Surgery Database, version 2.81 (http://www.sts.org/registries-researchcenter/sts-national-database/adult-cardiac-surgery-database/ data-collection).

The Northwell Health Institutional Review Board approved this study (\#17-0078; August 7, 2017); patient consent was waived.

\section{Statistical Analysis}

Study data were collected and managed using REDCap electronic data capture tools hosted at Lenox Hill Hospital [Harris 2009]. All statistical evaluation was performed using Statistical Package for the Social Sciences (SPSS) for Windows, version 26.0 (IBM Corp., Armonk, NY).

Analysis was undertaken using Fisher exact test or $\chi^{2}$ test for categorical variables, as appropriate. For continuous variables, Student's $t$ test was used for normally distributed variables, and Mann-Whitney $U$ test was used for those values with skewed distributions. Results were considered statistically significant at $P<.05$.

\section{RESULTS}

\section{Patient Population}

Of 152 consecutive patients who underwent repair of acute type A aortic dissection over the study period, 51 (33.6\%) underwent a full urine toxicology screen at presentation and

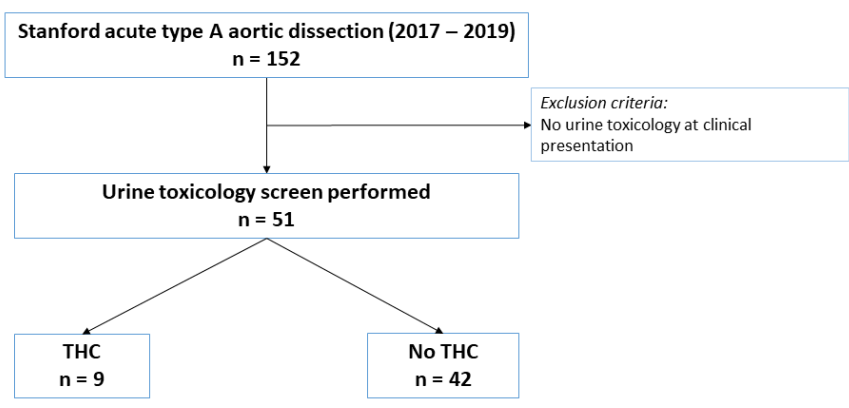

Figure 1. Patient flow diagram. THC indicates tetrahydrocannabinol. were thus eligible for inclusion in our analysis. Of these 51 patients, $9(17.6 \%)$ tested positive for marijuana, specifically THC (Figure 1). All THC patients were male, age $48 \pm 11.3$ years (mean \pm standard deviation), significantly younger than those who did not test positive for THC $(P=.004)$.

A greater proportion of patients in the THC cohort had a preexisting diagnosis of ascending aortic aneurysm compared with the no-THC group (44.4\% versus $4.8 \%, P=.006)$. Three patients in the THC group $(33.3 \%)$ also tested positive for recent cocaine use, and 3 others had toxicology evidence of amphetamine consumption. Patient demographics are summarized in Table 1 .

\section{Dissection Repair}

All patients underwent expeditious repair of acute dissection upon diagnosis, the details of which are outlined in Table 2. A detailed description of our surgical technique for type A dissection repair is beyond the scope of this discussion, but has been reported previously [Hemli 2017]. Selective unilateral antegrade cerebral perfusion during hypothermic circulatory arrest via the innominate artery was used in all cases.

Those patients who had negative THC results had longer cross-clamp times than the THC patients $(124.7 \pm 53.3$ versus $86.9 \pm 41.0$ minutes, $P=.05)$, and their perfusion times tended to be longer $(205.1 \pm 78.8$ versus $155.7 \pm 41.0$ minutes, $P=$ $.09)$, although the latter difference was not statistically significant. This finding is interesting, and somewhat difficult to explain, considering there was no significant difference in the types of aortic reconstructions undertaken between the 2 groups. There may have been a slight trend toward a greater proportion of no-THC patients undergoing somewhat more complex aortic procedures.

\section{Clinical Outcomes}

Thirty-day mortality for the entire cohort of 51 patients was $19.6 \%$ (10 deaths). The majority of patients who died were negative for THC. Of the 9 patients who were positive for THC, there was 1 death $(11.1 \%)$. This difference in short-term mortality did not reach statistical significance, and its clinical implications remain unclear. The incidence of major postoperative morbidity between the 2 groups was essentially comparable. Postoperative outcomes are outlined in Table 3.

\section{DISCUSSION}

Controversy about the potential risks of marijuana use has abounded for decades [Sznitman 2015]. The substance initially gained popularity in the 1960 s, when it was considered essentially harmless, although this perception was to completely reverse a decade later, when the agent was deemed "dangerous," first in the court of public opinion, and subsequently through political action [Carliner 2017]. Interestingly, beliefs about the relative safety of THC were to completely change again by $>40$ years later. California was the first state in the US to legalize medical marijuana [Hasin 2018]. As of January 2020, THC was legal in 11 states for recreational use and 33 
Table 1. Patient Demographics

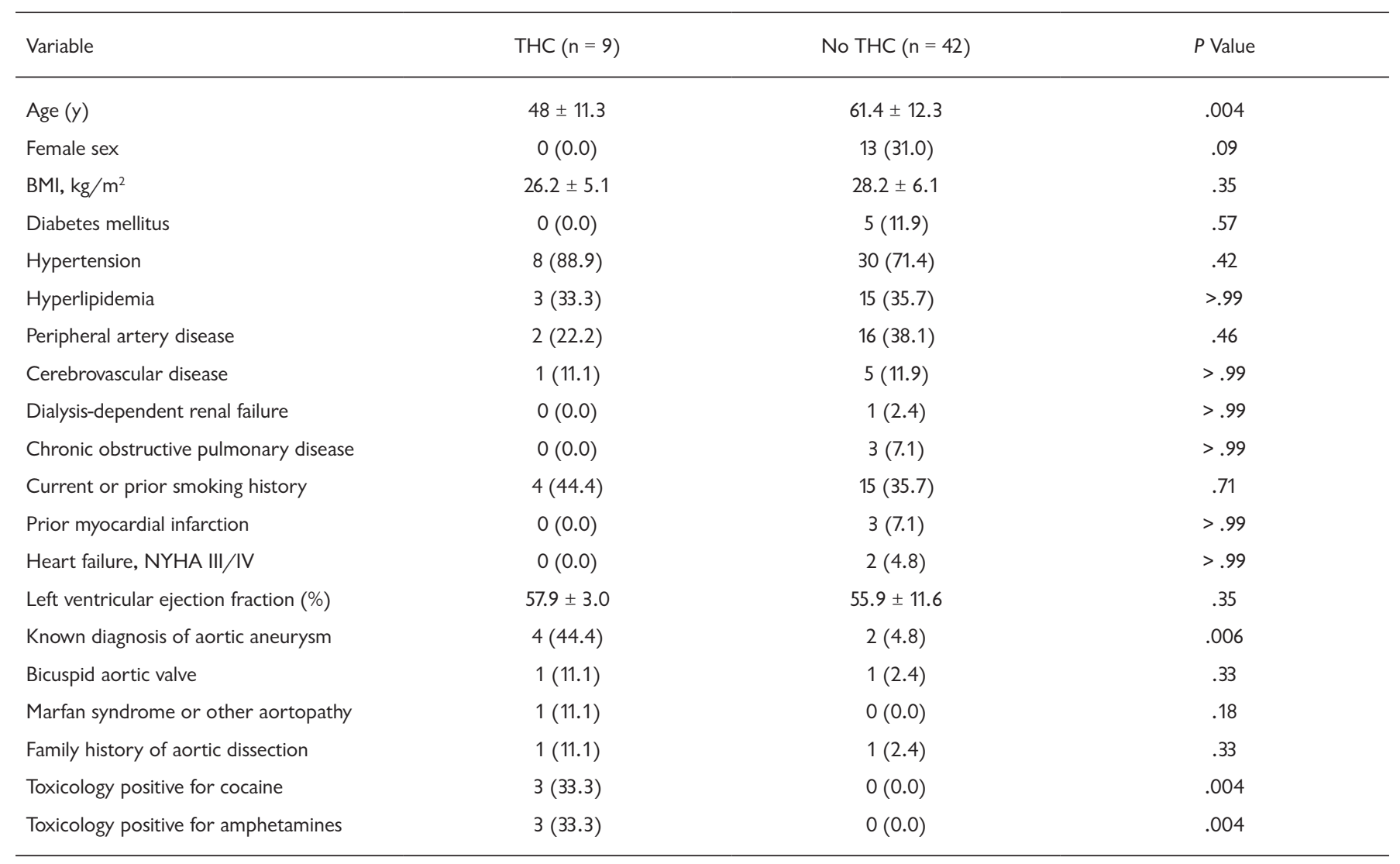

Values are mean \pm standard deviation or $n(\%)$.

BMI indicates body mass index; NYHA, New York Heart Association.

Table 2. Operative Data

\begin{tabular}{lccc}
\hline Variable & THC $(\mathrm{n}=9)$ & No THC $(\mathrm{n}=42)$ & $P$ Value \\
\hline Cardiopulmonary bypass time, mins & $155.7 \pm 73.6$ & $205.1 \pm 78.8$ & .09 \\
Aortic cross-clamp time $(\mathrm{min})$ & $86.9 \pm 41.0$ & $124.7 \pm 53.3$ & .05 \\
Hypothermic circulatory arrest time $(\mathrm{min})$ & $29.8 \pm 16.9$ & $29.4 \pm 18.8$ & .96 \\
Lowest intraoperative temperature $\left({ }^{\circ} \mathrm{C}\right)$ & $24.0 \pm 2.9$ & $22.1 \pm 4.3$ & .26 \\
Aortic procedure performed & & & $.25(59.5)$ \\
Isolated replacement of ascending aorta & $7(77.8)$ & $15(35.7)$ & .45 \\
Aortic root replacement & $1(11.1)$ & $2(4.8)$ & .45 \\
Total arch replacement & $1(11.1)$ & \\
\hline
\end{tabular}

Values are mean \pm standard deviation or $\mathrm{n}(\%)$. 
states for medicinal use [Zvonarev 2019]. State policymakers were forced to develop their own regulatory systems, since marijuana remains illegal under federal law [Kilmer 2017].

The growing consumption of marijuana created a parallel demand for a more potent product [Barry 2018]. On a national level, marijuana potency increased from $1.8 \%$ to $10.7 \%$ between 1990 and 2010 [Maxwell 2016]. The lack of universal guidelines for the regulation, production, and distribution of commercially legalized marijuana has been poorly addressed [Sevigny 2014]. Although the legalization of marijuana has afforded a number of potential societal economic benefits, such as generating a marketplace for creative enterprise and increasing taxation revenues [Hall 2019], its impacts on public health safety are not yet clearly known.

Marijuana remains classified as a Schedule I drug under the Controlled Substances Act, which defines it as having no medical purpose and a higher potential for abuse [Mead 2019]. It contains the chemicals THC and cannabidiol (CBD). Currently, the Food and Drug Administration (FDA) has approved 3 CBD medications (dronabinol, cannabidiol, and nabilone) for medicinal indications, including nausea but excluding analgesia [Oberbarnscheidt 2020]. Marijuana extracts that contain $<0.3 \%$ THC are excluded from the Schedule I classification and are readily available throughout the US as overthe-counter products in variable CBD potencies.

Non-FDA-approved marijuana products, such as those found in certain foods and oils, may contain potentially harmful substances, particularly in the form of synthetic marijuana [Chase 2016; Gunderson 2014]. It has been reported that adolescents tend to prefer the synthetic form of THC because it is often cheaper, is associated with a greater sense of euphoria after consumption, is less detectable using standard drug testing, and it may actually be misperceived as being legal, even though it is not [Palamar 2016]. Classifying marijuana as a controlled substance has made it somewhat more difficult to study the longer-term effects of its use and to formulate evidence-based regulation and policy-making [Martz 2018].

The adverse cardiovascular effects associated with marijuana use have largely been reported only through case reports or smaller observational studies. Based on these findings, clinicians are nevertheless encouraged to consider routine screening for marijuana when young adults present with cardiovascular symptoms [DeFilippis 2020]. Although marijuana is not considered to be a sympathomimetic agent per se, its impact on the cardiovascular system can be synergistic when combined with other stimulants, such as cocaine [Lukas 1994]. This is worth noting, given that 3 of the 9 patients in our series who tested positive for THC also had toxicology consistent with recent cocaine consumption, and 3 others had evidence of amphetamine use. Marijuana has been shown to increase heart rate, blood pressure, and systemic sympathetic tone. Consistent marijuana use has also been shown to increase carboxyhemoglobin levels, resulting in a relatively smaller systemic reservoir of oxygenated hemoglobin. This classic discrepancy between oxygen supply and demand is thought to be one of the mechanisms underlying marijuana-associated cardiac injury [Desbois 2013; Lindsay 2005; Mittlemen 2001].

Given that marijuana can potentiate sympathetic activity, particularly when used in conjunction with other stimulating agents, it would not be surprising to suggest that it may place an individual at higher risk for aortic dissection, particularly in those who may already be predisposed to the pathology for other reasons. Indeed, we found that a greater proportion of patients in the THC group had a preexisting diagnosis of ascending aortic aneurysm than those in the no-THC cohort.

Even though our sample size is quite small, our study nevertheless represents the largest series to date to specifically interrogate dissection patients with respect to marijuana consumption. Of 51 dissection patients, $17.6 \%$ tested positive for THC, a positivity rate markedly higher than

Table 3. Short-Term Clinical Outcomes

\begin{tabular}{lccc}
\hline Variable & THC $(\mathrm{n}=9)$ & No THC $(\mathrm{n}=42)$ & $9(21.4)$ \\
\hline 30-Day mortality & $1(11.1)$ & $12(28.6)$ & .67 \\
Stroke & $0(0.0)$ & $2(4.8)$ & .09 \\
Reoperation for bleeding & $0(0.0)$ & $0(0.0)$ & $7(16.7)$ \\
Deep sternal wound infection & $0(0.0)$ & $2(4.8)$ & $>.99$ \\
New dialysis-dependent renal failure & $1(11.1)$ & $244.2 \pm 246.1$ & .99 \\
Sepsis of any cause & $1(11.1)$ & $14.7 \pm 11.8$ & .99 \\
Duration of ICU stay (h) & $244.8 \pm 236.1$ & $10(7$ to 21$)$ \\
Length of postoperative hospital stay (d) & $14.7 \pm 9.3$ & $6(14.3)$ \\
Length of postoperative hospital stay (d) & $11(10$ to 16) & .54 \\
Readmission to hospital with 30 days (for any reason) & $0(0.0)$ & .58 \\
\hline
\end{tabular}

Values are mean \pm standard deviation, $\mathrm{n}(\%)$, or median (interquartile range).

ICU indicates intensive care unit. 
would be expected for the general population [SAMHSA 2019]. To the best of our knowledge, the potential association between marijuana use and aortic dissection has been examined by only 1 other group, who described a type A dissection in a 56-year-old man who smoked marijuana and had a family history of both aortic aneurysm and dissection [Mason 2019].

Our study has a number of limitations that need to be acknowledged. Our analysis is retrospective and largely observational in nature. Not all dissection patients underwent toxicology screening at presentation, such that an element of selection bias cannot be eliminated. Our study population is small, and consequently, it is difficult to draw broad-ranging conclusions from such an underpowered analysis.

The legalization of marijuana for both medical and recreational use has paved the way for greater adverse cardiovascular events related to its increased use. Although marijuana has already been associated with ischemic heart disease, we suggest that all of the elements are in place for it to potentially increase the risk for aortic dissection as well, particularly if the patient has a preexisting aortic aneurysm or uses other illicit sympathomimetic agents, such as cocaine or amphetamines. We suggest that clinicians should have a lower threshold for considering the diagnosis of acute aortic dissection in any younger patient presenting with chest pain or associated symptoms who has a history of recent marijuana consumption.

\section{ACKNOWLEDGEMENTS}

The authors are grateful to Karthik Seetharam, MD, Efstathia A Mihelis, MBA, and Sridhar Uttara, BS, for their assistance in data collection and analysis, and in facilitating the overall preparation of this manuscript. Derek R. Brinster, $\mathrm{MD}$, is a consultant for Terumo Aortic (Bolton Medical), Cook Medical, and W.L. Gore \& Associates.

\section{REFERENCES}

Barry RA, Glantz SA. Marijuana regulatory frameworks in four US states: An analysis against a public health standard. Am J Public Health 2018;108:914-923.

Carliner H, Brown QL, Sarvet AL, Hasin DS. Cannabis use, attitudes, and legal status in the U.S.: A review. Prev Med 2017;104:13-23.

Chase PB, Hawkins J, Mosier J, et al. Differential physiological and behavioral cues observed in individuals smoking botanical marijuana versus synthetic cannabinoid drugs. Clin Toxicol (Phila) 2016;54:14-19.

DeFilippis EM, Bajaj NS, Singh A, et al. Marijuana use in patients with cardiovascular disease. J Am Coll Cardiol 2020;75:320-332.

Desbois AC, Cacoub P. Cannabis-associated arterial disease. Ann Vasc Surg 2013;27:996-1005.

Eagle KA, Isselbacher EM, DeSanctis RW, International Registry for Aortic Dissection (IRAD) Investigators. Cocaine-related aortic dissection in perspective. Circulation 2002;105:1529-1530.
Gunderson EW, Haughey HM, Ait-Daoud N, Joshi AS, Hart CL. A survey of synthetic cannabinoid consumption by current cannabis users. Subst Abus 2014;35:184-189.

Hall W, Stjepanović D, Caulkins J, et al. Public health implications of legalising the production and sale of cannabis for medicinal and recreational use. Lancet 2019;394:1580-1590.

Harris PA, Taylor R, Thielke R, et al. Research electronic data capture (REDCap)-A metadata-driven methodology and workflow process for providing translational research informatics support. J Biomed Inform 2009;42:377-381.

Hasin DS. US epidemiology of cannabis use and associated problems. Neuropsychopharmacology 2018;43:195-212.

Hemli JM, DeLaney ER, Dholakia KR, et al. Rethinking the paradigm: Modern approach to proximal aortic reconstruction demonstrates excellent outcomes. Heart Surg Forum 2017;20:E092-E097.

Kilmer B. Recreational cannabis-Minimizing the health risks from legalization. N Engl J Med 2017;376:705-707.

Lindsay AC, Foale RA, Warren O, Henry JA. Cannabis as a precipitant of cardiovascular emergencies. Int J Cardiol 2005;104:230-232.

Lukas SE, Sholar M, Kouri E, Fukuzako H, Mendelson JH. Marihuana smoking increases plasma cocaine levels and subjective reports of euphoria in male volunteers. Pharmacol Biochem Behav 1994;48:715-721.

Martz G, Tankersley W, Mekala HM, Motiwala H. Rates of synthetic cannabinoid use in adolescents admitted to a treatment facility. Prim Care Companion CNS Disord 2018;20:17m02265.

Mason EK, Gak AE, Finno JG, Cannon RD, Jacoby JL. Thoracic aortic dissection associated with marijuana use. J Emerg Med 2019;57:235-237.

Maxwell JC, Mendelson B. What do we know now about the impact of the laws related to marijuana? J Addict Med 2016;10:3-12.

Mead A. Legal and regulatory issues governing cannabis and cannabisderived products in the United States. Front Plant Sci 2019;10:697.

Mittlemen MA, Lewis RA, Maclure M, Sherwood JB, Muller JE. Triggering myocardial infarction by marijuana. Circulation 2001;103:2805-2809.

Oberbarnscheidt T, Miller NS. The impact of cannabidiol on psychiatric and medical conditions. J Clin Med Res 2020;12:393-403.

Palamar JJ, Barratt MJ. Synthetic cannabinoids: Undesirable alternatives to natural marijuana. Am J Drug Alcohol Abuse 2016;42:371-373.

ProCon.org. Legal Medical Marijuana States and DC. 2020. Available at https://medicalmarijuana.procon.org/legal-medical-marijuana-statesand-dc/ (Accessed January 27, 2021).

Richards JR, Bing ML, Moulin AK, et al. Cannabis use and acute coronary syndrome. Clin Toxicol 2019;57:831-841.

SAMHSA (Substance Abuse and Mental Health Services Administration). Key substance use and mental health indicators in the United States: Results from the 2018 National Survey on Drug Use and Health (HHS Publication No. PEP19-5068, NSDUH Series H-54). 2019. Rockville, MD: Center for Behavioral Health Statistics and Quality, Substance Abuse and Mental Health Services Administration. Available at: http://store.samhsa.gov/product/key-substance-use-and-mental-healthindicators-in-the-united-states-results-from-the-2018-national-surveyon-Drug-Use-and-Health/PEP19-5068 (Accessed January 27, 2021).

Sevigny EL, Pacula RL, Heaton P. The effects of medical marijuana laws on potency. Int J Drug Policy 2014;25:308-319.

Shah R, Berzingi C, Fan THM, Askari R, Khan MR. Cocaine-induced 
acute aortic dissection. J Emerg Med 2015;49:e87-e89.

Sznitman SR, Bretteville-Jensen AL. Public opinion and medical cannabis policies: Examining the role of underlying beliefs and national medical cannabis policies. Harm Reduct J 2015;12:46.
Wako E, LeDoux D, Mitsumori L, Aldea GS. The emerging epidemic of methamphetamine-induced aortic dissections. J Card Surg 2007;22:390-393.

Zvonarev V, Fatuki TA, Tregubenko P. The public health concerns of marijuana legalization: An overview of current trends. Cureus 2019;11:e5806. 